\title{
Small Molecules Targeting the Hedgehog Pathway: From Phenotype to Mechanistic Understanding
}

\author{
Sascha Hoogendoorn*
}

\begin{abstract}
Since the beginning of 2019, the Hoogendoorn lab is active at the University of Geneva. We are a Chemical Biology lab and our research focuses on the Hedgehog $(\mathrm{Hh})$ signalling pathway and the primary cilium, a small cellular organelle which corrects structure and function, is required to conduct the Hh signal. Ciliary Hh signalling plays an important role in embryonic development, and its dysregulation consequently results in developmental disorders as well as a variety of cancers. We use an interdisciplinary approach, ranging from organic chemistry to cell biology and genetics, to develop chemical tools to study and perturb ciliary signalling. In this account, I will highlight existing small molecules that target the Hh pathway, our efforts to discover new compounds, and the methodologies that we employ for target deconvolution and mechanism of action studies.
\end{abstract}

Keywords: Chemical Biology · Hedgehog signalling · Phenotypic screens · Small-molecule probes · Target identification

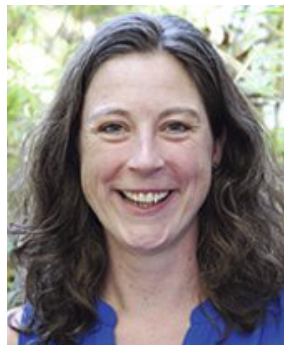

Sascha Hoogendoorn studied Chemistry at Leiden University, the Netherlands, where she also received her $\mathrm{PhD}$ under supervision of Prof. Herman Overkleeft in 2014. She subsequently joined the group of Prof. James Chen at Stanford University, USA, with a Rubicon postdoctoral fellowship (NWO). In 2019, she started her lab as a tenure-track assistant professor in Chemical Biology at the University of Geneva. Her interdisciplinary research program combines organic chemistry with cell biology and genetics to develop molecules that enable further dissection and manipulation of ciliary signalling.

\section{Introduction}

Cells respond to cues from their environment by activating a variety of intracellular signalling pathways, typically initiated by upstream receptor engagement and resulting in downstream transcriptional regulation of target genes. In the context of a developing organism, cell signalling ensures the correct formation and spatial restriction of tissues and organs, and is therefore of essential importance. One of the major developmental signalling pathways is the Hedgehog $(\mathrm{Hh})$ pathway. The Hh pathway was first discovered in the fruit fly Drosophila melanogaster where mutation of a single Hh gene leads to embryos with a 'spikey' appearance - hence the name Hedgehog. ${ }^{[1]}$ The Hh pathway has attracted a lot of attention in past years because of its obvious role in development, but also in stem cell maintenance and tissue homeostasis. ${ }^{[2]}$ An additional layer of complexity is added to the Hh pathway by its dependence on a specialized cellular organelle, the primary cilium. ${ }^{[3,4]}$ Furthermore, dysregulated Hh signalling contributes to the onset and progression of a variety of cancers, and small-molecule inhibitors of this pathway have proven clinical use. ${ }^{[5]}$ The Hoogendoorn lab at the University of Geneva is part of the Department of Organic Chemistry as well as the National

${ }^{*}$ Correspondence: Prof. S. Hoogendoorn, E-mail: sascha.hoogendoorn@unige.ch University of Geneva, Department of Organic Chemistry, NCCR Chemical Biology, Quai Ernest-Ansermet 30, CH-1211 Gèneve, Switzerland
Centre of Competence in Research (NCCR) Chemical Biology and in our research program we combine organic chemistry, cell biology, and genetics to decipher and modulate the molecular mechanisms underlying the Hh signalling pathway. Here, I will present an overview of small molecules currently available as therapeutics and tools that target the Hh pathway, and our efforts to identify novel chemotypes through phenotypic screening. We aim to tackle the challenge of going from a compound-induced phenotype to mechanistic understanding of its action through an interdisciplinary approach, to ultimately arrive at a toolbox of well-defined compounds to unravel Hh pathway biology.

\section{The Hedgehog Signalling Pathway and the Primary Cilium - Balancing Health and Disease States}

The primary cilium is a microtubule-based organelle that protrudes from the cell and is roughly $3-5 \mu \mathrm{m}$ in length. Most mammalian cells only have one, non-motile, cilium; its presence is dynamically regulated by cell cycle stage as ciliogenesis is initiated by the docking of the centrioles to the cell membrane, forming the basal body. ${ }^{[6]}$ Genetic mutations leading to lack of cilia or defects in ciliary function underlie a broad range of developmental diseases, collectively called ciliopathies. Clinical manifestations of ciliopathies include mental retardation, obesity, digital anomalies, retinal degeneration, skeletal dysplasias, cerebral anomalies and renal disease. ${ }^{[7]}$ In many cases, ciliopathy phenotypes can be related back to dysregulation of the Hh pathway. All the core $\mathrm{Hh}$ pathway components dynamically localize to the primary cilium, which thus functions as an 'organizing center' (Fig. 1). In the off-state of the pathway (Fig. 1A), the Hedgehog protein receptor Patched1 (PTCH1) localizes to cilia and inhibits the main activator Smoothened (SMO) by restricting its localization to the cytoplasm. The transcription factors GLI2 and GLI3 are bound to the negative regulator Suppressor of Fused (SUFU), phosphorylated by protein kinase A (PKA), and proteolytically processed to their N-terminal repressor forms. Upon pathway activation by Hedgehog protein binding to PTCH1, PTCH1 moves out and SMO moves into the cilium. It is incompletely understood what triggers the localization changes and which endogenous small molecules are responsible for the communication between PTCH1 and SMO, though recent evidence points toward cholesterol and 
sterol analogues. ${ }^{[8]}$ Activation of SMO leads to a shift in the transcriptional state of the GLI proteins. Upon SMO activation, the interaction between GLI proteins and SUFU is lost. SUFU, GLI2 and GLI3 all accumulate at the ciliary tip during the initial stage of signal transduction and this is required in order for the GLI proteins to become transcriptionally active. ${ }^{[9-12]}$ Consequently, the formation of the activator forms of the GLI proteins, GLI-A, leads to the transcription of Hh target genes, including Glil (the most downstream transcriptional activator - a positive feedback loop) and Ptchl (a negative feedback loop) (Fig. 1B). ${ }^{[13,14]}$
A: no HH: pathway off

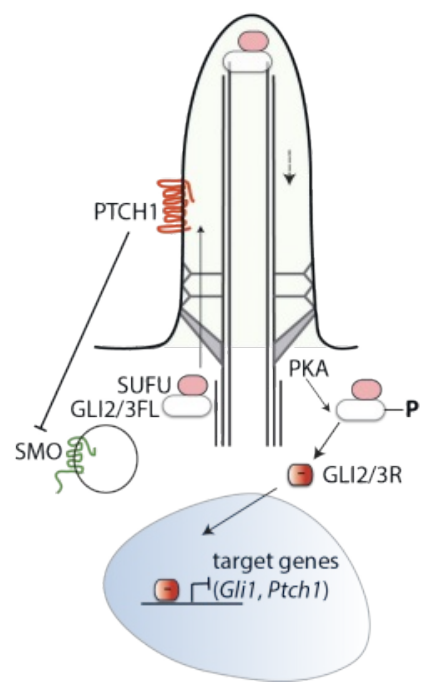

B: $+\mathrm{HH}$ : pathway on

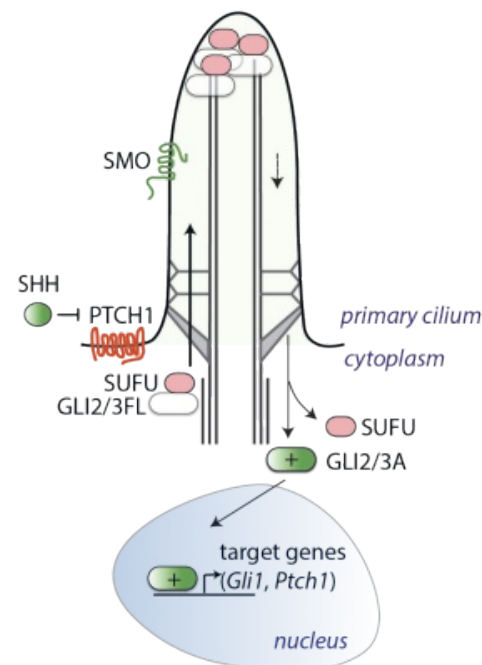

Fig. 1. Schematic representation of the Hedgehog pathway. A) In the offstate, PTCH1 inhibits SMO translocation to the cilium. The GLI proteins are phosphorylated by PKA and processed into their transcriptional repressor forms (GLI2/3R). B) Upon binding of $\mathrm{SHH}, \mathrm{PTCH} 1$ is inhibited. SMO enters the cilium leading to the formation of GLI activators (GLI2/3A) through decreased cAMP levels and inhibition of PKA.

The main players involved in signal transduction have been the subject of extensive research efforts over the past years and are generally well characterized. ${ }^{[13]}$ However, on a mechanistic level many gaps remain in our knowledge of ciliary signalling. During my postdoctoral period at Stanford, my co-workers and I envisioned a novel approach to systematically identify genes involved in cilium assembly and Hh signalling. We developed a CRISPR/ Cas9-based screening platform for ciliary signalling and conducted a genome-wide knockout screen to identify genes important for Hh signal transduction and cilium function. Among the many hits we retrieved were known core Hh pathway components, genes involved in cilia assembly, and ciliopathy genes with very few false positives or false negatives, corroborating the importance of the primary cilium in Hh signal transduction. Follow-up studies on six hit genes with uncharacterized roles in ciliary $\mathrm{Hh}$ signalling led to the discovery of novel cilium components, candidate ciliopathy genes, and a complex required for centriole stability. ${ }^{[15]}$ Currently, we are investigating the importance of several of the screen hits in more detail, to learn more about their role in cilium biology, and as a starting point for target-based drug development. Furthermore, we aim to repurpose and extend this screening platform for mechanism of action studies of Hh signalling inhibitors, as detailed in Section 4.3.

\section{Small Molecule Inhibitors of Ciliary Hedgehog Signalling}

\subsection{Smoothened Inhibitors}

Uncontrolled GLI activation contributes to the onset and/or progression of a number of (paediatric) cancers, including medul- loblastoma, advanced basal cell carcinoma, rhabdomyosarcoma, meningioma, and small cell lung cancer. Mechanisms include enhanced paracrine (i.e. stroma to tumour) and autocrine Hh signalling, or oncogenic mutations in or loss of pathway components such as Ptchl or Sufu. ${ }^{[5,16]}$ Clearly, small molecules acting on this pathway could be of great therapeutic benefit, but for a long time the druggability of the pathway remained elusive. A breakthrough came with the discovery, using a chemical biology approach, that $\mathrm{SMO}$ is the cellular target of the natural product cyclopamine (Fig. 2). ${ }^{[17]}$ After years of research, cyclopamine had been identified as the responsible teratogen present in the corn lily Veratrum californicum. When this plant was consumed by pregnant ewes, their lambs presented with severe developmental defects, with cyclopia as the most striking manifestation. ${ }^{[18]}$ Many drug discovery programs were subsequently launched in order to find novel SMO inhibitors that could be used in the treatment of $\mathrm{Hh}$ pathway-driven cancers (Fig. 2). Indeed, this resulted in FDA approval of vismodegib for treatment of advanced basal cell carcinoma in 2012, which was allowed on the Swiss market in 2013. Initial results with vismodegib were extremely promising, with great reduction in tumour burden. Upon prolonged treatment, however, tumour relapse occurred in many cases. This resistance is induced by oncogenic mutations in SMO or downstream pathway components. [19-21] Furthermore, SMO inhibitors are inherently ineffective to tumours driven by mutations in downstream components (e.g. SUFU mutations) or non-canonical GLI-signalling (activation of GLI not induced by activation of SMO, e.g. via Ras/mitogen-activated protein kinase (MAPK) or phosphoinositide 3-kinase (PI3K)/Akt signalling $\left.{ }^{[22-24]}\right)$. Regardless of their promise and pitfalls as therapeutics, SMO inhibitors have been, and still are, instrumental in advancing SMO biology, by providing a means to temporally and differentially control SMO localization and structure. ${ }^{[25-27]}$
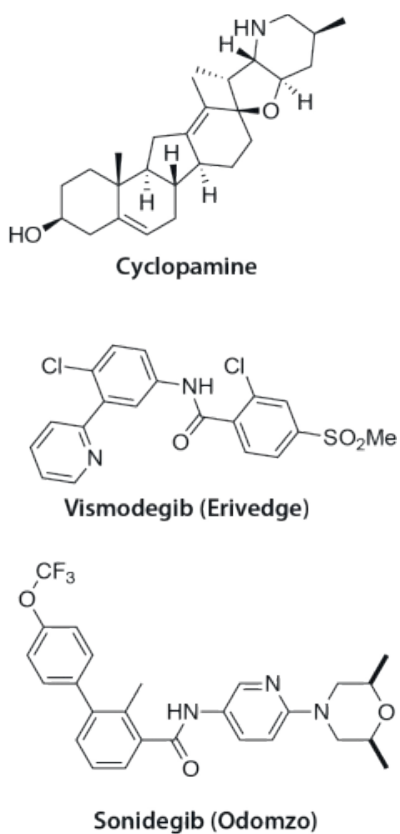

Fig. 2. SMO inhibitors. The discovery that the natural product cyclopamine binds the transmembrane protein SMO spurred the development of the SMO-targeting clinical drugs vismodegib and sonidegib.

\subsection{Non-Smoothened Hh Pathway Inhibitors}

The majority of compounds acting on the Hh pathway are SMO inhibitors, and it is clear that this GPCR-like protein is the most druggable in the Hh pathway. However, to overcome the inherent limitations of SMO inhibitors, it is imperative to discover compounds that act downstream of SMO. A variety of $\mathrm{Hh}$ pathway inhibitors has appeared in the literature that do not bind 
to SMO (a selection is shown in Fig. 3). These inhibitors prevent GLI activation, either directly, such as robotnikinin that binds Sonic hedgehog protein, ${ }^{[28]}$ or through indirect mechanisms. For example, forskolin inhibits Hh signalling through activation of adenylate cyclases and PKA, ${ }^{[29]}$ BET bromodomain inhibitors such as JQ1 and I-BET151 disrupt signalling through transcriptional regulation of GLI1, ${ }^{[30,31]}$ and epigenetic drugs targeting HDAC prevent deacetylation of GLI1[32] (Fig. 3A). Other published GLI antagonists include arsenic trioxide, ${ }^{[33]}$ itraconazole, ${ }^{[34]}$ arcyriaflavins, ${ }^{[35]}$ physalins, ${ }^{[36]}$ arylisoquinolones, ${ }^{[37]}$ and mercaptobenzoimidazoles, ${ }^{[38]}$ but none of these have a validated cellular target and/or mechanism of action, diminishing their potential as tool compounds or therapeutic leads (Fig. 3B).

\subsection{Phenotypic Screens for Hh Pathway Inhibitor Discovery}

High-throughput phenotypic screening is a powerful method to assess the biological activity of a large collection of molecules. As there is no a priori target-bias, phenotypic screening allows for the discovery of molecules with alternative mechanisms to those already described. Not surprisingly, the Hh pathway has been central to various industrial and academic phenotypic screening campaigns to identify additional inhibitors (Table 1). ${ }^{[39-46]}$ Most screens relied on the use of a transcriptional reporter, containing an 8xGLI-binding motif and a minimal promoter, followed by the firefly luciferase gene ('LIGHT'). ${ }^{[47]}$ Cells are stimulated with ShhN (the N-terminal domain of SHH)-conditioned medium 'ShhN' or a synthetic SMO agonist (SAG, Ag1.5 or purmorphamine ${ }^{[48]}$ ) to activate the pathway at the level of PTCH1 or SMO, respectively. There is a limited number of cell lines that can be used for this purpose, because few cells express all the necessary components for Hh signal transduction in culture. Fibroblasts, including the mouse cell line NIH-3T3, are the model system of choice because of their robust ciliogenesis and $\mathrm{Hh}$ signalling properties. Additionally, mouse Leydig TM3 cells have been used successfully. ${ }^{[44]}$ In some cases, the target of the hit compound was successfully identified, yielding very useful tool compounds. A good example of this is HPI-4 (Fig. 3A). Originally found in a screen by Hyman et al., treatment of cells with this compound resulted in shorter than usual cilia, so this class of molecules was renamed 'ciliobrevins' ${ }^{[40]}$ Follow-up studies showed that ciliobrevins bind the motor protein dynein, thereby disrupting intraflagellar transport (IFT) in the cilium and inhibiting Hh signalling. ${ }^{[49,50]}$ Ciliobrevins have subsequently been successfully employed as tools to study dynein motor function in a number of studies. ${ }^{[51]}$

Despite the promise of Hh pathway and ciliogenesis inhibitors for therapeutic purposes and as tool compounds to examine the biology associated with their cellular targets, few inhibitors with favourable properties have been found to date. Moreover, most known inhibitors lack a defined target and mechanism, thus not reaching their full potential. We are collaborating with the ACCESS screening facility (NCCR Chemical Biology) to conduct a high-content, microscopy-based screen, using a chemically diverse library of compounds and cells that express fluorescent protein-tagged $\mathrm{Hh}$ pathway and ciliary proteins. In luciferasebased transcriptional screening the cells are lysed in the process, and information on cell and cilium morphology is lost. In contrast, our screening approach allows the identification of novel molecules that perturb signal transduction and/or the primary cilium, while toxic compounds can be directly excluded.

\section{Hit-to-Lead: How to Identify Cellular Targets and Mechanism of Action of Small Molecules?}

The fact that so few hits that came out of phenotypic screens have been properly characterized in terms of their target and mode of action illustrates the underlying problem: it is usually challenging and time-consuming to identify the target and the mechanism by which an inhibitor acts if there is no other information besides the phenotype available. The problem requires an interdisciplinary approach, since no one strategy guarantees a successful outcome

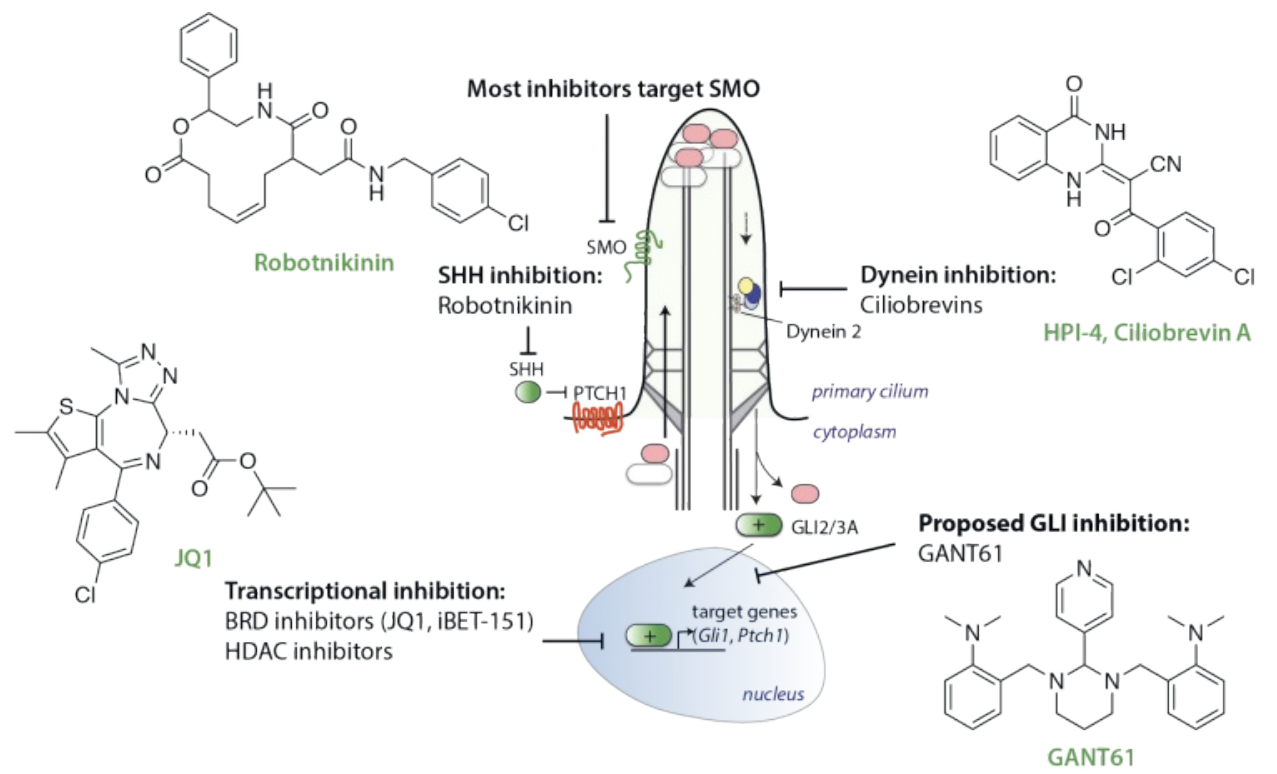

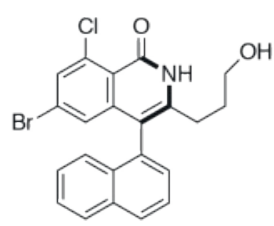

4-arylisoquinolone $2 \mathrm{~m}$
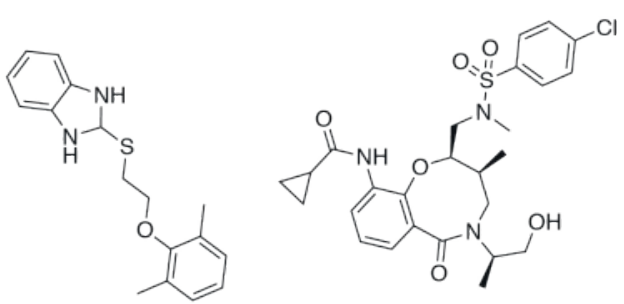

Fig. 3. Selection of Hedgehog signalling inhibitors. A) Inhibitors have very diverse targets and modes of action. Compounds shown in green have validated targets. Robotnikinin inhibits Sonic Hedgehog (SHH). ${ }^{[28]} \mathrm{HPI}-$ 4 (Ciliobrevin A) is an inhibitor of cytoplasmic dynein. ${ }^{[40,49]} \mathrm{JQ1}$ inhibits BRD4 resulting in transcriptional inhibition of GLI proteins. ${ }^{[30]}$ GANT61 prevents GLI1 DNA-binding. ${ }^{[4]}$ B) Inhibitors with unknown target. HPI-1 was identified in a large-scale phenotypic screen conducted by the Chen lab and has been validated as a downstream inhibitor but has an unknown target. ${ }^{[40]}$ BRD50837 and 4-arylisoquinolone $2 \mathrm{~m}$ do not directly engage SMO, but their mechanism is unknown. ${ }^{[37,39]}$ No information on the target is available for mercaptobenzoimidazole 10a. ${ }^{[38]}$ 
Table 1. Hedgehog inhibitors discovered through phentotypic screening

\begin{tabular}{|l|l|l|l|l|l|l|l|l|}
\hline compound & cell line & activation by & inhibitors of & readout & target & comments \\
\hline HPI-1, 2, 3, 4 & Shh-LIGHT2 & SAG & Hh pathway & luminescence & $\begin{array}{l}\text { HPI-1, 2, 3: } \\
\text { unknown } \\
\text { HPI-4: dynein }\end{array}$ \\
SMO
\end{tabular}

for each molecule under examination. A structured pipeline for target deconvolution of Hh pathway inhibitors is lacking to date, and one of the goals of our group is to change this - we are developing methodologies ranging from probe synthesis to CRISPR-based screening, taking advantage of the interdisciplinarity of our group.

\subsection{Photo-affinity Labelling}

Photo-affinity labelling has proven to be a valuable strategy in the identification of cellular target(s) of small molecules (Fig. 4). It relies on the physical proximity of the ligand to its target when bound, and the ability of photoactive organic groups such as diazirine, aryl azide, and benzophenone to react with light to form highly reactive intermediates that can crosslink to nearby protein residues. Once the ligand is covalently bound to its target, the complex can be visualized or extracted from the proteome for downstream analysis using a reporter group (biotin, fluorophore) (Fig. 4A). Typically, this involves the introduction of both a photo-crosslinking group and a reporter group to the pharmacophore core of a hit compound. ${ }^{[52,53]}$ It is essential that the probe behaves as much as possible as the parent molecule to be able to extrapolate the information gained with the probe to the actual hit compound. Hits from phenotypic screens are typically moderately potent, with potencies in the $1-10 \mu \mathrm{M}$ range. Extensive structure-activity relationship (SAR) studies are often necessary to sample the chemical space surrounding the pharmacophore, yielding 1) analogues with increased potency and selectivity, tremendously increasing the utility of the initial hit, and 2) guidelines for where to incorporate the photoactive and reporter groups without destroying its potency. In some cases, the reporter group can be incorporated in the probe (through use of a linker) directly. When the bulk of the reporter group changes the potency, localization or selectivity of the probe compared to the parent compound, a bio-orthogonal ligation strategy can be used (Fig. 4B). Over the past years, a variety of bio-orthogonal reactions have been developed, most notably 1,3-dipolar cycloadditions (both $\mathrm{Cu}(\mathrm{I})$-mediated and strain-promoted click reactions) and inverse electron demand Diels-Alder reactions as these require small functional groups (azides, alkynes, cyclopropene).[54]
In some cases, it can be advantageous to combine the ligation handle and the photo-labile group in an 'all-in-one' functional group, i.e. 'clickable' aliphatic diazirines. ${ }^{[55]}$ Cells are incubated with the probe after which the photoactive group is activated by irradiation, resulting in a covalent adduct between the probe and its protein target. Subsequently, the ligation handle is used to attach an affinity tag (biotin) for pull-down and mass spectrometry experiments, a fluorophore for visualization of the target, either by gel electrophoresis or fluorescence microscopy, or both by incorporation of a biotin-fluorophore construct (Fig. 4C). ${ }^{[56]} \mathrm{A}$ drawback of photo-affinity labelling studies for target identificaation (ID) is the potential of retrieving abundant, 'sticky' proteins that bind non-specifically. Competition experiments with unlabelled analogues are useful to differentiate between specific and nonspecific binding. Crosslinked samples with and without excess competitor can be resolved by $1 \mathrm{D}$ gel electrophoresis followed by streptavidin-based detection of biotin, or by 2D FITGE (fluorescence difference in two-dimensional gel electrophoresis) through labelling with different fluorophores. Spots that are exclusively labelled by probe alone are most likely to be specific and can be cut out of the gel and processed for downstream mass spectrometry (Fig. 4C).[57] Although SAR studies and probe development are labour-intensive, a distinct advantage of this methodology is that once the target is elucidated, the probe can directly be employed in cellular assays to visualize its target in various contexts. For example, the probe BODIPY-cyclopamine, a fluorescent cyclopamine analogue, is widely used in competition assays to assess if a small-molecule Hh pathway inhibitor binds to SMO. $[17,40,45,58]$

\subsection{Mass Spectrometry-based Methods}

Given the constraints of SAR studies and the development of photo-crosslinking probes, label-free approaches for target deconvolution are valuable alternatives. Recent advances in quantitative mass spectrometry-based proteomics have sparked the development of global proteome profiling methodologies for target deconvolution. Thermal proteome profiling is a mass spectrometry-based extension of the cellular thermal shift (CETSA). [59,60] CETSA relies on the stabilization of a protein target upon 
A Photo-crosslink groups<smiles>FC(F)(F)C1(c2ccccc2)CN=N1</smiles>

Trifluoromethylphenyldiazirine

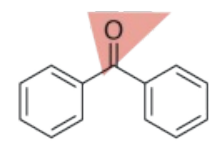

Benzophenone
B

Reporter groups

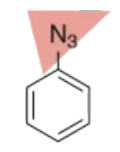

Phenylazide

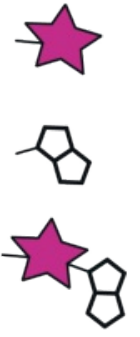

fluorophore: visualization of the probe in cells, in-gel fluorescence

biotin: pull-down with streptavidin

fluorophore - biotin: both visualization and pull-down

C
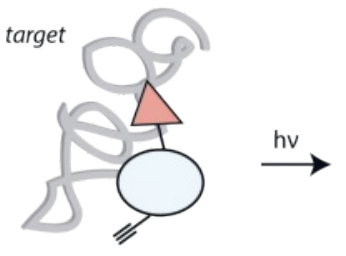

Target engagement
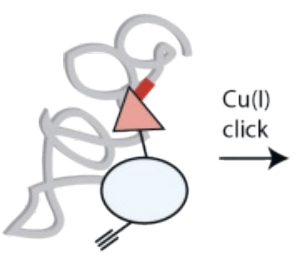

Probe covalenty cross-linked to the target

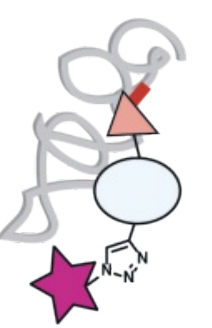

SAR studies

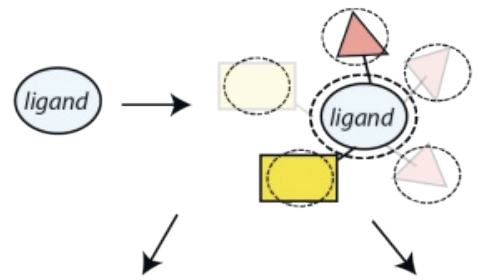

One-step probe

Two-step probe

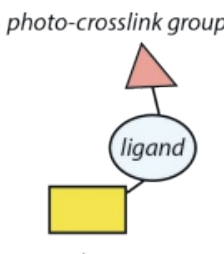

reporter group

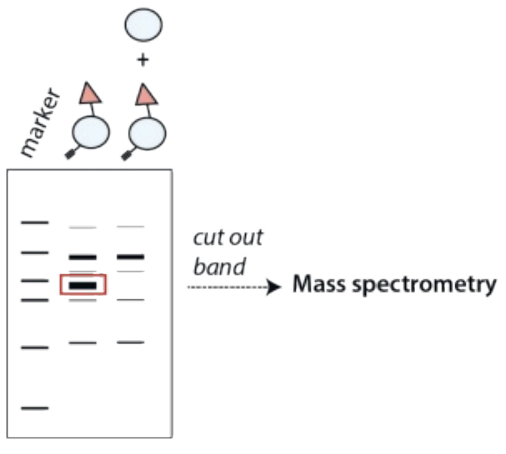

ligation handle for

bio-orthogonal labeling

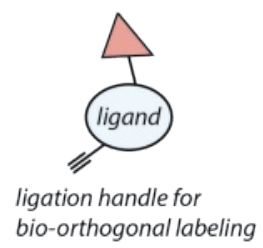

Target ID by pull-down/purification (biotin)

or visualization (fluorophore)

Fig. 4. Target deconvolution by photo-affinity labelling. A) Examples of photo-crosslink groups and reporter groups used in photo-affinity probes. B) Starting from a screen hit, SAR studies are done to determine where the ligand can be functionalized with a photo-crosslink and reporter group. Depending on the bulk that can be added to the ligand, a one-step or two-step probe can be synthesized. C) In a typical photo-affinity experiment, the cells are incubated with the probe (here shown is a two-step probe), which is covalently crosslinked to its target upon exposure to light. Next, a reporter group is attached through bio-orthogonal labelling. The labelled proteins are then pulled-down using streptavidin beads or resolved on SDS-PAGE. By comparing the labelled proteins with and without competitor, specific bands can be distinguished from aspecific background, and subjected to mass spectrometry analysis to determine the identity of the corresponding protein.

binding of a small molecule, resulting in a shift in its melting temperature. Cells are treated with the compound or DMSO control and subjected to increasing temperatures before lysis and collection of the soluble (non-denatured) fraction. In thermal proteome profiling, the soluble fractions are then analysed by mass spectrometry, giving global, quantitative proteome profiles. Proteins that are (de)stabilized upon compound treatment are then likely direct or indirect targets of the compound. The advantage of this methodology is that, if successful, it will provide both the cellular target as well as information on the mechanism through indirect targets. Furthermore, it allows the identification of offtargets or multiple targets if the phenotype arises through polypharmacologic action of the compound. The drawback however is that it is hard to detect low abundance proteins, so its success largely depends on the identity(ies) of the protein target. ${ }^{[59,61,62]}$ A slightly different approach is that of limited proteolysis, where the conformational changes induced by compound binding result in differential proteolytic patterns, which can be detected by mass spectrometry when comparing conditions with and without compound. ${ }^{[63]}$

\subsection{Genetic Screening Approaches}

Instead of studying the physical association of the drug and its protein target, genetic screening can be used to perturb protein levels and thereby also the ability of the compound to exert its effect. With the discovery of CRISPR-mediated gene editing, pooled screening approaches have gained great momentum, with many successful examples in diverse areas of biology over the past couple of years. [64-66] Fewer examples exist where genetic screening has been employed to study compound action, even though this should in principle be a very powerful alternative strategy to proteomics-based approaches. [67,68] To identify the target of a cytotoxic compound, a growthbased screen is a viable option, as illustrated by the identification of NAMPT as the cellular target of the anti-cancer drug STF-118804. ${ }^{[69]}$ Genetic screens also provide information on potential resistance mechanisms to drugs or conceivable combination therapies. For non-cytotoxic drugs either FACS-based screening approaches can be used, or a growth phenotype can be engineered. ${ }^{[15,70]}$ At Stanford, I have previously developed a cell line compatible with growthbased pooled CRISPR-screening for Hh signalling. These cells are engineered to contain a blasticidin resistance marker under control of the GLI-driven promoter (Fig. 5A). Hence, under signalling conditions the cells become resistant to the antibiotic blasticidin, but when signal transduction is inhibited, either through addition of a compound or knock-out of a positive regulator of the pathway, the cells die when exposed to blasticidin. Conversely, knock-out of a negative regulator such as $S u f u$ results in (constitutive) increased blasticidin resistance (Fig. 5B). ${ }^{[15]}$ We now repurpose this screening platform with a focused library of sgRNA's to assess which genes' knockout or knockdown results in compound hypersensitivity or 
resistance (Fig. 5C). Depending on where the compound acts in the pathway (downstream or upstream), it will be sensitive to modulation of a different set of genes. For example, we found that knockout of adenylate cyclase 6 results in resistance to the Smoothened inhibitor vismodegib, while it has no effect on the action of HPI-1 (a downstream inhibitor with unknown target). By using a diverse set of sgRNAs targeting genes important for Hedgehog signalling and cilium structure and function, 'gene-activity profiles' can be generated to gain systems-level information on compound action. The advantage of using focused-libraries is that it drastically reduces the number of cells that are needed to get significant results such that multiple compounds can be essayed and directly compared in one screen. While target identification through genetic screens is also possible, the use of focused libraries might preclude this - there is no way of knowing a priori if the target is present in the library.

\section{Concluding Remarks}

Each of the different methods for target ID and mechanism of action studies has limitations and distinct advantages. None of these methods is likely to provide a single target or definitive mechanism, but rather lists of potential compound interactors. The complementary information yielded through different approaches will however greatly facilitate the generation of rational hypotheses and follow-up studies. Well-validated small-molecules are very useful to study a biological system, as they provide a layer of temporal control that is difficult to obtain by genetic methods. Compounds can be dosed to titrate the effects, they can be washed out, and they can be combined with chemical or genetic perturbations to study additive effects. This is especially true for a complex signalling network such as the Hedgehog pathway. A collection of compounds with known targets, ranging from upstream Smoothened to downstream GLI transcription factors, and defined mode of action would be of tremendous benefit to unravel the molecular mechanisms of $\mathrm{Hh}$ signal transduction and we believe that our chemical biology program can contribute towards this goal. We are currently employing photo-affinity labelling and genetic approaches to study Hh pathway inhibitors without validated target and mechanism, and hope to extend our studies to mass spectrometry methods in the near future.

\section{Acknowledgements}

I'd like to thank my mentors and colleagues for their support, and the members of my group for their enthusiasm and dedication. Our work is financially supported by the National Centre of Competence in Research (NCCR) Chemical Biology and the Swiss National Science Foundation (project grant 189246).

Received: June 15, 2020

[1] C. Nüsslein-Volhard, E. Wieschaus, Nature 1980, 287, 795

[2] J. Briscoe, P. P. Therond, Nature Rev. Mol. Cell Biol. 2013, 14, 416
A

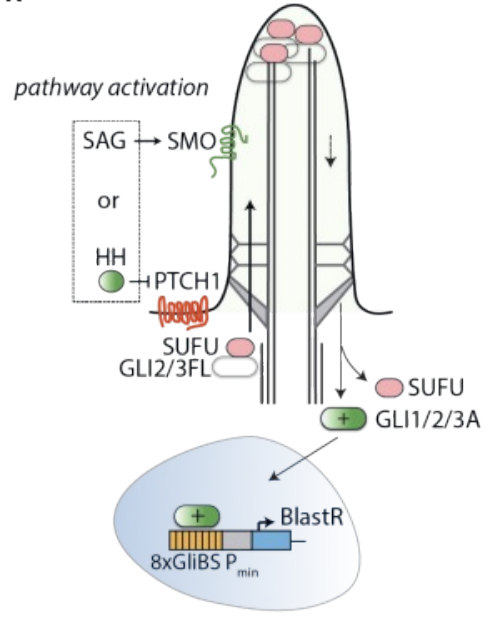

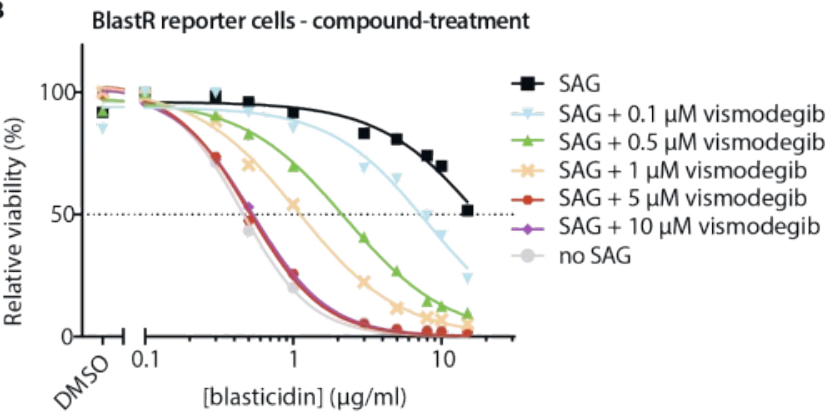

BlastR reporter cells with Cas9 - knockout of Hh pathway control genes

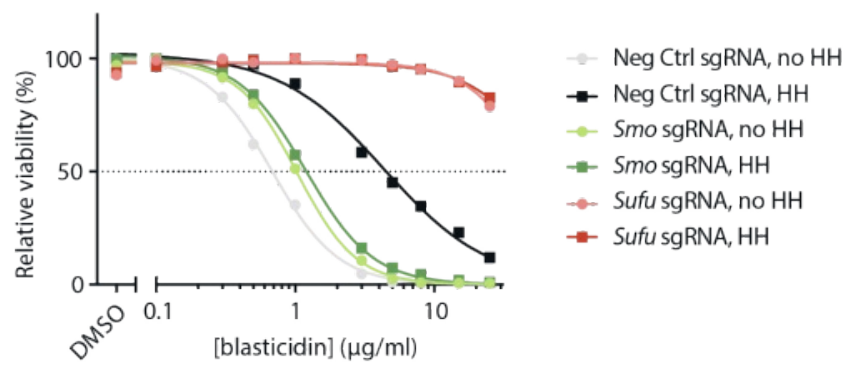

c

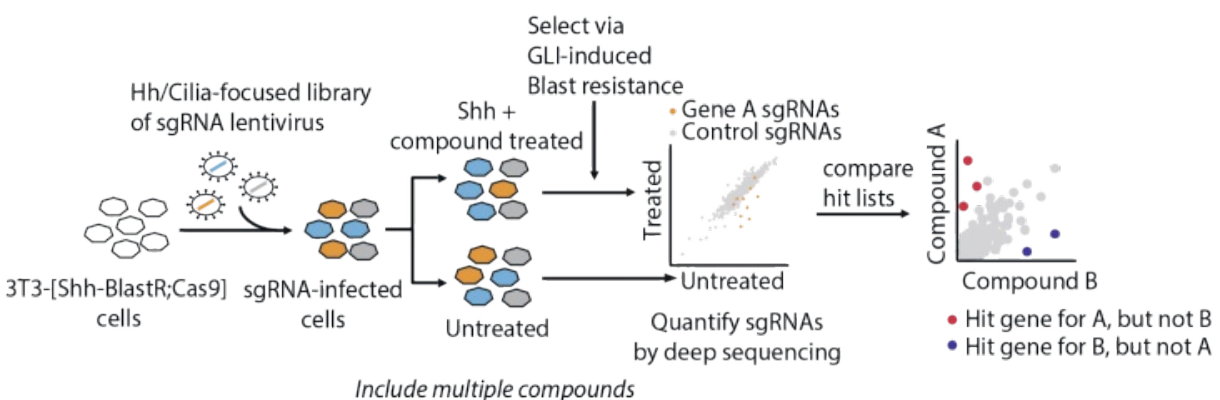

Fig. 5. Genetic screens for mechanism of action studies. A) To convert active Hedgehog signalling into a growth phenoptype, we have engineered an NIH3T3 cell line that contains a blasticidin resistance gene (BlastR) under control of a GLI promotor. Upon stimulation with HH protein or the synthetic SMO agonist SAG, the cells become resistant to the antibiotic blasticidin. $\left.{ }^{[15]} \mathrm{B}\right)$ Pharmacological inhibition of Hh signalling by vismodegib, or genetic knock-out of Smo results in decreased cell viability in the presence of blasticidin, in a dose-dependent fashion. Conversely, constitutively active Sufu knockout cells are much more resistant to blasticidin than control cells treated with $\mathrm{HH}$. C) Schematic representation of a focused genetic screen in combination with small-molecule modulators of $\mathrm{Hh}$ signalling, resulting in different genetic profiles depending on which genes are important for a compounds' action. 
[3] D. Huangfu, A. Liu, A. S. Rakeman, N. S. Murcia, L. Niswander, K. V. Anderson, Nature 2003, 426, 83

[4] F. Bangs, K. V. Anderson, Cold Spring Harb. Perspect. Biol. 2017, 9:a028217

[5] L. L. Rubin, F. J. de Sauvage, Nat. Rev. Drug Discov. 2006, 5, 1026

[6] S. C. Goetz, K. V. Anderson, Nat. Rev. Genet. 2010, 11, 331

[7] F. Hildebrandt, T. Benzing, N. Katsanis, N. Engl. J. Med. 2011, 364, 1533

[8] Y. Zhang, D. P. Bulkley, Y. Xin, K. J. Roberts, D. E. Asarnow, A. Sharma, B. R. Myers, W. Cho, Y. Cheng, P. A. Beachy, Cell 2018, 175, 1352

[9] C. J. Haycraft, B. Banizs, Y. Aydin-Son, Q. Zhang, E. J. Michaud, B. K. Yoder, PLoS Genet. 2005, 1, e53

[10] J. Kim, M. Kato, P. A. Beachy, Proc. Natl Acad. Sci. USA 2009, 106, 21666

[11] N. Santos, J. F. Reiter, J. Cell Sci. 2014, 127, 1500

[12] M.-H. Chen, C. W. Wilson, Y.-J. Li, K. K. L. Law, C.-S. Lu, R. Gacayan, X. Zhang, C. Hui, P.-T. Chuang, Gene Dev. 2009, 23, 1910

[13] E. Pak, R. A. Segal, Dev. Cell 2016, 38, 333

[14] F. Wu, Y. Zhang, B. Sun, A. P. McMahon, Y. Wang, Cell Chem. Biol. 2017, 24,252

[15] D. K. Breslow, S. Hoogendoorn, A. R. Kopp, D. W. Morgens, B. K. Vu, M. C. Kennedy, K. Han, A. Li, G. T. Hess, M. C. Bassik, J. K. Chen, M. V. Nachury, Nat. Genetics 2018, 50, 460

[16] L. V. Goodrich, L. Milenković, K. M. Higgins, M. P. Scott, Science 1997, 277, 1109

[17] J. K. Chen, J. Taipale, M. K. Cooper, P. A. Beachy, Gene Dev. 2002, 16, 2743

[18] J. K. Chen, Nat. Prod. Rep. 2016, 33, 595

[19] G. J. Dijkgraaf, B. Alicke, L. Weinmann, T. Januario, K. West, Z. Modrusan, D. Burdick, R. Goldsmith, K. Robarge, D. Sutherlin, S. J. Scales, S. E. Gould, R. L. Yauch, F. J. de Sauvage, Cancer Res. 2011, 71, 435

[20] K. D. Robarge, S. A. Brunton, G. M. Castanedo, Y. Cui, M. S. Dina, R. Goldsmith, S. E. Gould, O. Guichert, J. L. Gunzner, J. Halladay, W. Jia, C. Khojasteh, M. F. Koehler, K. Kotkow, H. La, R. L. Lalonde, K. Lau, L. Lee, D. Marshall, J. C. Marsters Jr., L. J. Murray, C. Qian, L. L. Rubin, L. Salphati, M. S. Stanley, J. H. Stibbard, D. P. Sutherlin, S. Ubhayaker, S. Wang, S. Wong, M. Xie, Bioorg. Med. Chem. Lett. 2009, 19, 5576

[21] C. M. Rudin, C. L. Hann, J. Laterra, R. L. Yauch, C. A. Callahan, L. Fu, T. Holcomb, J. Stinson, S. E. Gould, B. Coleman, P. M. LoRusso, D. D. Von Hoff, F. J. de Sauvage, J. A. Low, N. Engl. J. Med. 2009, 361, 1173

[22] N. A. Riobo, G. M. Haines, C. P. Emerson Jr., Cancer Res. 2006, 66, 839

[23] N. A. Riobo, K. Lu, X. Ai, G. M. Haines, C. P. Emerson Jr., Proc. Natl Acad. Sci. USA 2006, 103, 4505

[24] F. Aberger, A. Ruiz i Altaba, Semin. Cell Dev. Biol. 2014, 33, 93

[25] S. Nachtergaele, D. M. Whalen, L. K. Mydock, Z. Zhao, T. Malinauskas, K. Krishnan, P. W. Ingham, D. F. Covey, C. Siebold, R. Rohatgi, eLife 2013, 2 , e 01340

[26] C. Wang, H. Wu, T. Evron, E. Vardy, G. W. Han, X.-P. Huang, S. J. Hufeisen, T. J. Mangano, D. J. Urban, V. Katritch, V. Cherezov, M. G. Caron, B. L. Roth, R. C. Stevens, Nat. Commun. 2014, 5, 4355

[27] R. Rohatgi, L. Milenkovic, R. B. Corcoran, M. P. Scott, Proc. Natl Acad. Sci. USA 2009, 106, 3196

[28] B. Z. Stanton, L. F. Peng, N. Maloof, K. Nakai, X. Wang, J. L. Duffner, K. M. Taveras, J. M. Hyman, S. W. Lee, A. N. Koehler, J. K. Chen, J. L. Fox, A. Mandinova, S. L. Schreiber, Nat. Chem. Biol. 2009, 5, 154

[29] D. U. Mick, R. B. Rodrigues, R. D. Leib, C. M. Adams, A. S. Chien, S. P. Gygi, M. V. Nachury, Dev. Cell 2015, 35, 497

[30] Y. Tang, S. Gholamin, S. Schubert, M. I. Willardson, A. Lee, P. Bandopadhayay, G. Bergthold, S. Masoud, B. Nguyen, N. Vue, B. Balansay, F. Yu, S. Oh, P. Woo, S. Chen, A. Ponnuswami, M. Monje, S. X. Atwood, R. J. Whitson, S. Mitra, S. H. Cheshier, J. Qi, R. Beroukhim, J. Y. Tang, R. Wechsler-Reya, A. E. Oro, B. A. Link, J. E. Bradner, Y.-J. Cho, Nat. Med. 2014, 20, 732

[31] J. Long, B. Li, J. Rodriguez-Blanco, C. Pastori, C.-H. Volmar, C. Wahlestedt, A. Capobianco, F. Bai, X.-H. Pei, N. G. Ayad, D. J. Robbins, J. Biol. Chem. 2014, 289, 35494

[32] P. K. Dhanyamraju, P. S. Holz, F. Finkernagel, V. Fendrich, M. Lauth, Mol. Cancer Ther. 2015, 14, 727

[33] J. Kim, J. J. Lee, J. Kim, D. Gardner, P. A. Beachy, Proc. Natl Acad. Sci. USA 2010, 107, 13432

[34] J. Kim, J. Y. Tang, R. Gong, J. Kim, J. J. Lee, K. V. Clemons, C. R. Chong, K. S. Chang, M. Fereshteh, D. Gardner, T. Reya, J. O. Liu, E. H. Epstein, D. A. Stevens, P. A. Beachy, Cancer Cell 2010, 17, 388

[35] T. Hosoya, M. A. Arai, T. Koyano, T. Kowithayakorn, M. Ishibashi, Chembiochem 2008, 9, 1082

[36] M. A. Arai, K. Uchida, S. K. Sadhu, F. Ahmed, M. Ishibashi, Beilstein J. Org. Chem. 2014, 10

[37] G. Shan, J. Flegel, H. Li, C. Merten, S. Ziegler, A. P. Antonchick, H. Waldmann, Angew. Chem. Int. Ed. 2018, 57, 14250

[38] S. Gräßle, S. Susanto, S. Sievers, E. Tavsan, M. Nieger, N. Jung, S. Bräse, ACS Med. Chem. Lett. 2017, 8, 931

[39] G. I. Schaefer, J. R. Perez, J. R. Duvall, B. Z. Stanton, A. F. Shamji, S. L. Schreiber, J. Am. Chem. Soc. 2013, 135, 9675

[40] J. M. Hyman, A. J. Firestone, V. M. Heine, Y. Zhao, C. A. Ocasio, K. Han, M. Sun, P. G. Rack, S. Sinha, J. J. Wu, D. E. Solow-Cordero, J. Jiang, D. H. Rowitch, J. K. Chen, Proc. Natl Acad. Sci. USA 2009, 106, 14132
[41] M. Lauth, A. Bergström, T. Shimokawa, R. Toftgård, Proc. Natl Acad. Sci. USA 2007, 104, 8455

[42] V. M. Wu, S. C. Chen, M. R. Arkin, J. F. Reiter, Proc. Natl Acad. Sci. USA 2012, 109, 13644

[43] C. H. Williams, J. E. Hempel, J. Hao, A. Y. Frist, M. M. Williams, J. T. Fleming, G. A. Sulikowski, M. K. Cooper, C. Chiang, C. C. Hong, Cell Rep. 2015, 11, 43

[44] F. Bassilana, A. Carlson, J. A. DaSilva, B. Grosshans, S. Vidal, V. Beck, B. Wilmeringwetter, L. A. Llamas, T. B. Showalter, P. Rigollier, A. Bourret, A. Ramamurthy, X. Wu, F. Harbinski, S. Plonsky, L. Lee, H. Ruffner, P. Grandi, M. Schirle, J. Jenkins, A. W. Sailer, T. Bouwmeester, J. A. Porter, V. Myer, P. M. Finan, J. A. Tallarico, J. F. Kelleher Iii, K. Seuwen, R. K. Jain, S. J. Luchansky, Nat. Chem. Biol. 2014, 10, 343

[45] L. Kremer, C. Schultz-Fademrecht, M. Baumann, P. Habenberger, A. Choidas, B. Klebl, S. Kordes, H. R. Schöler, J. Sterneckert, S. Ziegler, G. Schneider, H. Waldmann, Angew. Chem. Int. Ed. 2017, 56, 13021

[46] L. Kremer, E. Hennes, A. Brause, A. Ursu, L. Robke, H. T. Matsubayashi, Y. Nihongaki, J. Flegel, I. Mejdrová, J. Eickhoff, M. Baumann, R. Nencka, P. Janning, S. Kordes, H. R. Schöler, J. Sterneckert, T. Inoue, S. Ziegler, H. Waldmann, Angew. Chem. Int. Ed. 2019, 58, 16617

[47] H. Sasaki, C. Hui, M. Nakafuku, H. Kondoh, Development 1997, 124, 1313

[48] T. J. Carney, P. W. Ingham, BMC Biology 2013, 11, 37

[49] A. J. Firestone, J. S. Weinger, M. Maldonado, K. Barlan, L. D. Langston, M. O'Donnell, V. I. Gelfand, T. M. Kapoor, J. K. Chen, Nature 2012, 484, 125

[50] S. K. See, S. Hoogendoorn, A. H. Chung, F. Ye, J. B. Steinman, T. SakataKato, R. M. Miller, T. Cupido, R. Zalyte, A. P. Carter, M. V. Nachury, T. M. Kapoor, J. K. Chen, ACS Chem. Biol. 2016, 11, 53

[51] D. H. Roossien, K. E. Miller, G. Gallo, Front. Cell Neurosci. 2015, 9, 252

[52] E. Smith, I. Collins, Future Med. Chem. 2015, 7, 159

[53] D. J. Lapinsky, Bioorg. Med. Chem. 2012, 20, 6237

[54] L. I. Willems, W. A. van der Linden, N. Li, K.-Y. Li, N. Liu, S. Hoogendoorn, G. A. van der Marel, B. I. Florea, H. S. Overkleeft, Acc. Chem. Res. 2011 44,718

[55] S.-S. Ge, B. Chen, Y.-Y. Wu, Q.-S. Long, Y.-L. Zhao, P.-Y. Wang, S. Yang, RSC Adv. 2018, 8, 29428

[56] W. P. Heal, B. Jovanovic, S. Bessin, M. H. Wright, A. I. Magee, E. W. Tate, Chem. Commun. 2011, 47, 4081

[57] J. Park, S. Oh, S. B. Park, Angew. Chem. Int. Ed. 2012, 51, 5447

[58] P. Kozielewicz, C.-F. Bowin, A. Turku, G. Schulte, Mol. Pharmacol. 2020, 97, 23

[59] M. M. Savitski, F. B. M. Reinhard, H. Franken, T. Werner, M. F. Savitski, D. Eberhard, D. M. Molina, R. Jafari, R. B. Dovega, S. Klaeger, B. Kuster, P. Nordlund, M. Bantscheff, G. Drewes, Science 2014, 346, 1255784

[60] D. M. Molina, R. Jafari, M. Ignatushchenko, T. Seki, E. A. Larsson, C. Dan, L. Sreekumar, Y. Cao, P. Nordlund, Science 2013, 341, 84

[61] M. M. Savitski, N. Zinn, M. Faelth-Savitski, D. Poeckel, S. Gade, I. Becher, M. Muelbaier, A. J. Wagner, K. Strohmer, T. Werner, S. Melchert, M. Petretich, A. Rutkowska, J. Vappiani, H. Franken, M. Steidel, G. M. Sweetman, O. Gilan, E. Y. N. Lam, M. A. Dawson, R. K. Prinjha, P. Grandi, G. Bergamini, M. Bantscheff, Cell 2018, 173, 260

[62] F. B. M. Reinhard, D. Eberhard, T. Werner, H. Franken, D. Childs, C. Doce, M. F. Savitski, W. Huber, M. Bantscheff, M. M. Savitski, G. Drewes, Nat. Methods 2015, 12, 1129

[63] B. Lomenick, R. Hao, N. Jonai, R. M. Chin, M. Aghajan, S. Warburton, J. Wang, R. P. Wu, F. Gomez, J. A. Loo, J. A. Wohlschlegel, T. M. Vondriska, J. Pelletier, H. R. Herschman, J. Clardy, C. F. Clarke, J. Huang, Curr. Protoc. Chem. Biol. 2009, 106, 21984

[64] H. Koike-Yusa, Y. Li, E. P. Tan, C. Velasco-Herrera Mdel, K. Yusa, Nat. Biotechnol. 2014, 32, 267

[65] O. Shalem, N. E. Sanjana, E. Hartenian, X. Shi, D. A. Scott, T. S. Mikkelsen, D. Heckl, B. L. Ebert, D. E. Root, J. G. Doench, F. Zhang, Science 2014, 343, 84

[66] T. Wang, J. J. Wei, D. M. Sabatini, E. S. Lander, Science 2014, 343, 80

[67] M. Kampmann, Chem. Commun. 2017, 53, 7162

[68] R. M. Deans, D. W. Morgens, A. Ökesli, S. Pillay, M. A. Horlbeck, M. Kampmann, L. A. Gilbert, A. Li, R. Mateo, M. Smith, J. S. Glenn, J. E. Carette, C. Khosla, M. C. Bassik, Nat. Chem. Biol. 2016, 12, 361

[69] C. J. Matheny, M. C. Wei, M. C. Bassik, A. J. Donnelly, M. Kampmann, M. Iwasaki, O. Piloto, D. E. Solow-Cordero, D. M. Bouley, R. Rau, P. Brown, M. T. McManus, J. S. Weissman, M. L. Cleary, Chem. Biol. 2013, 20, 1352

[70] G. V. Pusapati, J. H. Kong, B. B. Patel, A. Krishnan, A. Sagner, M. Kinnebrew, J. Briscoe, L. Aravind, R. Rohatgi, Dev. Cell 2018, 44, 113.

\section{License and Terms}

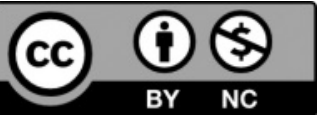

This is an Open Access article under the terms of the Creative Commons Attribution License CC BY_NC 4.0. The material may not be used for commercial purposes.

The license is subject to the CHIMIA terms and conditions: (http:// chimia.ch/component/sppagebuilder/?view=page\&id=12).

The definitive version of this article is the electronic one that can be found at https://doi.org/10.2533/chimia.2020.652 